\title{
The Prognostic Role of Cancer Stem Cells in Breast Tumors
}

\author{
Maurizio Di Bonito ${ }^{a}$, Monica Cantile ${ }^{\mathrm{a}, \mathrm{b}}$, Gabriella Malzone ${ }^{\mathrm{a}}$, \\ Giuseppina Liguoria ${ }^{a}$, Gerardo Botti ${ }^{\mathrm{a}}$
}

Recent acquisitions on human carcinogenesis suggest that small populations of tumor stem cells can influence and modify neoplastic cells behavior and aggressiveness, as well as therapeutic response. Breast cancer is a complex disease which encompasses a wide range of phenotypes with different histopathological, clinical and molecular features.

It is therefore extremely important to identify the unit of tumor cells which lead the progression of the disease in order to set the optimal therapy for each breast cancer subtype. Late studies advice that breast cancer ability to proliferate, progress and spread is based on a limited subpopulation of cells with properties similar to stem cells, which has led to coin the term "breast cancer stem cells" (BC$\mathrm{SCs}$ ) [1]. The presence of BCSCs niches results in a poor sensitivity to current chemotherapy treatments. Therefore, the residual chemoresistant tumor stem cells would be able, after therapy, to fuel the development of a new tumor mass [2].

Several stemness markers have been described for the various histological subtypes of breast cancer, among them CD44, CD24, CD133, EpCAM, CD166, Lgr5, CD47, ALDH1 and the most recent ABCG2.

Trans-membrane proteins CD44 and CD24 are among the most studied BCSC markers.

High levels of CD44 associated to low levels of CD24 $(\mathrm{CD} 44(+) / \mathrm{CD} 24(-/$ low $))$ would characterize stem populations in breast cancer [3].

Trans-differentiation of normal breast cells with CD44low/ CD24+ phenotype, in BCSCs with CD44(+)/CD24(-/low) phenotype, is achieved after Ras oncogene activation along with TERT over-expression [4]. Either the absence or low expression of CD24 associated with CD44 high expression in BCSCs is related to an invasive phenotype, poor prognosis and low survival $[5,6]$.

Aldehyde dehydrogenase-1 (ALDH1) is a newly proposed marker for BCSCs identification.

ALDH1 high expression represents a marker for breast, lung,

Manuscript accepted for publication February 19, 2013

aPathology Unit, Istituto Nazionale Tumori Fondazione "G. Pascale", via Mariano Semmola 80131, Napoli, Italy

${ }^{\mathrm{b}}$ Corresponding author: Monica Cantile, Pathology Unit, National Cancer Institute, Pascale Hospital, via Mariano Semmola 80131,

Naples, Italy. Email: monicantile@libero. it

doi: http://dx.doi.org/10.4021/jocmr1302w prostate, liver, neck and colon CSCs [7]. Specifically, ALDH1 expression in breast cancer accounts only for $20-25 \%$. Of this percentage, an average of $5 \%$ of cells is positive to ALDH1. High positivity in tumor cells is associated with high histological grade, ERBB2 over-expression, absence of hormone receptors ER and PgR and worse prognosis $[8,9]$.

Recent and numerous studies show that positivity for CD133 allows to identify CSCs in breast cancer [10]. CD133 is expressed by several solid tumors, including invasive breast cancer triple negative, with very low levels of expression compared to other CSCs markers previously reported, like CD44 and ALDH1 [11]. In early-onset breast cancers, associated with mutations on BRCA1, CD133+ cells show CSCs properties [10]. The employment of this tumor stemness marker in breast cancers has become popular more recently and its expression is often described as associated with a worse prognosis $[12,13]$.

ABCG2, or Breast Cancer Resistance Protein (BCRP), recognizes and carries several conventional anti-tumor drugs, including small chemotherapy molecules. Numerous lines of evidence suggest that ABCG2 can be associated with the presence of tumor stem cells and correlates with chemoresistence and a poor prognosis in several human neoplasias [14]. ABCG2 was over-expressed in human hematopoietic stem cells (HSCs) and it proved to be a marker of stem cell populations in different organs, including pancreas, lung, testis, brain and prostate [15]. Its role in breast cancer is still fairly discussed, although recent lines of evidence have indicated ABCG2 as a potential stemness marker also in this neoplasia [16]. However, there are no important recommendations on its prognostic role in this malignancy.

The role of these markers in breast cancer progression is not clear yet and above all no markers have been defined yet as the most adequate for the characterization of the niches of tumor stem cells.

Likely, several combinations of these markers would allow a more appropriate characterization of CSCs in different breast tumor subtypes.

It should be also ascertained those most closely related to prognosis and to therapeutic treatment resistance. So, it could be possible to delineate a more correct stratification of patients at risk, speculating also to directly interfere with the activity of these molecules [17], as is the case in other malignancies [18, 19], in order to establish more personalized therapeutic strategies.

\section{References}

1. Liu S, Dontu G, Wicha MS. Mammary stem cells, self- 
renewal pathways, and carcinogenesis. Breast Cancer Res. 2005;7(3):86-95.

2. Chuthapisith S, Eremin J, El-Sheemey M, Eremin O. Breast cancer chemoresistance: emerging importance of cancer stem cells. Surg Oncol. 2010;19(1):27-32.

3. Ponti D, Costa A, Zaffaroni N, Pratesi G, Petrangolini G, Coradini D, Pilotti S, et al. Isolation and in vitro propagation of tumorigenic breast cancer cells with stem/progenitor cell properties. Cancer Res. 2005;65(13):55065511.

4. Paranjape AN, Mandal T, Mukherjee G, Kumar MV, Sengupta K, Rangarajan A. Introduction of SV40ER and hTERT into mammospheres generates breast cancer cells with stem cell properties. Oncogene. 2012;31(15):18961909.

5. Ahmed MA, Aleskandarany MA, Rakha EA, Moustafa RZ, Benhasouna A, Nolan C, Green AR, et al. A CD44()/CD24(+) phenotype is a poor prognostic marker in early invasive breast cancer. Breast Cancer Res Treat. 2012;133(3):979-995.

6. Idowu MO, Kmieciak M, Dumur C, Burton RS, Grimes MM, Powers CN, Manjili MH. CD44(+)/CD24(-/low) cancer stem/progenitor cells are more abundant in triple-negative invasive breast carcinoma phenotype and are associated with poor outcome. Hum Pathol. 2012;43(3):364-373

7. Douville J, Beaulieu R, Balicki D. ALDH1 as a functional marker of cancer stem and progenitor cells. Stem Cells Dev. 2009;18(1):17-25.

8. Sakakibara M, Fujimori T, Miyoshi T, Nagashima T, Fujimoto H, Suzuki HT, Ohki Y, et al. Aldehyde dehydrogenase 1-positive cells in axillary lymph node metastases after chemotherapy as a prognostic factor in patients with lymph node-positive breast cancer. Cancer. 2012;118(16):3899-3910.

9. Ohi Y, Umekita Y, Yoshioka T, Souda M, Rai Y, Sagara Y, Tanimoto A. Aldehyde dehydrogenase 1 expression predicts poor prognosis in triple-negative breast cancer.
Histopathology. 2011;59(4):776-780.

10. Wright MH, Calcagno AM, Salcido CD, Carlson MD, Ambudkar SV, Varticovski L. Brca1 breast tumors contain distinct CD44+/CD24- and CD133+ cells with cancer stem cell characteristics. Breast Cancer Res. 2008;10(1):R10.

11. Wu Y, Wu PY. CD133 as a marker for cancer stem cells: progresses and concerns. Stem Cells Dev. 2009;18(8):1127-1134.

12. Zhao P, Lu Y, Jiang X, Li X. Clinicopathological significance and prognostic value of CD133 expression in triple-negative breast carcinoma. Cancer Sci. 2011;102(5):1107-1111.

13. Ieni A, Giuffre G, Adamo V, Tuccari G. Prognostic impact of CD133 immunoexpression in node-negative invasive breast carcinomas. Anticancer Res. 2011;31(4):13151320.

14. Crea F, Danesi R, Farrar WL. Cancer stem cell epigenetics and chemoresistance. Epigenomics. 2009;1(1):63-79.

15. Ding XW, Wu JH, Jiang CP. ABCG2: a potential marker of stem cells and novel target in stem cell and cancer therapy. Life Sci. 2010;86(17-18):631-637.

16. Britton KM, Eyre R, Harvey IJ, Stemke-Hale K, Browell D, Lennard TW, Meeson AP. Breast cancer, side population cells and ABCG2 expression. Cancer Lett. 2012;323(1):97-105.

17. Gangopadhyay S, Nandy A, Hor P, Mukhopadhyay A. Breast cancer stem cells: a novel therapeutic target. Clin Breast Cancer. 2013;13(1):7-15.

18. Rappa G, Fodstad O, Lorico A. The stem cell-associated antigen CD133 (Prominin-1) is a molecular therapeutic target for metastatic melanoma. Stem Cells. 2008;26(12):3008-3017.

19. Smith LM, Nesterova A, Ryan MC, Duniho S, Jonas M, Anderson M, Zabinski RF, et al. CD133/prominin-1 is a potential therapeutic target for antibody-drug conjugates in hepatocellular and gastric cancers. Br J Cancer. 2008;99(1):100-109. 